\title{
ON THE COEFFICIENTS OF MULTIPLE SERIES WITH RESPECT TO VILENKIN SYSTEM
}

\author{
Valentin A. Skvortsov — Francesco Tulone
}

\begin{abstract}
We give a sufficient condition for coefficients of double series $\sum \sum_{n, m} a_{n, m} \chi_{n, m}$ with respect to Vilenkin system to be convergent to zero when $n+m \rightarrow \infty$. This result can be applied to the problem of recovering coefficients of a Vilenkin series from its sum.
\end{abstract}

\section{Introduction}

It is easy to give an example showing that the rectangular convergence almost everywhere of a double Walsh series

$$
\sum_{n=0}^{\infty} \sum_{m=0}^{\infty} a_{n, m} w_{n}(x) w_{m}(y)
$$

does not imply in general that the coefficients $a_{n, m}$ tend to zero when $n+m \rightarrow \infty$. It suffices to take a non-trivial Walsh series $\sum_{n=0}^{\infty} a_{n} w_{n}(x)$ convergent to zero almost everywhere on [0,1] (see [2] or [4]) and to put $a_{n, m}=a_{n}$ for all $m$.

However, a simple sufficient condition to guarantee that the coefficients $a_{n, m}$ of (1.1) tend to zero when $n+m \rightarrow \infty$ was given in [5].

In this paper, we generalize the above theorem to the case of the Vilenkin system (see [1]). Although we are proving the result for the two-dimensional case, the same method can be used to obtain a similar result for any dimension. We present also some application of this result to the problem of recovering coefficients of a Vilenkin series from its sum (more details on this application will be given in [10]).

(C) 2017 Mathematical Institute, Slovak Academy of Sciences. 2010 Mathematics Subject Classification: 42C10, 26 A39.

Keywords: Vilenkin system, multiple Vilenkin series, rectangular convergence, Perron integral, Saks continuity, recovering the coefficients.

Supported by RFBR (grant no. 17-01-00286). 


\section{VALENTIN A. SKVORTSOV — FRANCESCO TULONE}

\section{Preliminaries}

Let

$$
\mathcal{P}=\left\{p_{j}\right\}_{j=0}^{\infty}
$$

be a fixed sequence of natural numbers with $p_{j} \geq 2$ for $j=0,1,2, \ldots$ Set $m_{0}=1$ and $m_{k}=p_{0} \cdot p_{1} \cdot \ldots \cdot p_{k-1}$.

For each $x \in[0,1)$ we consider its $\mathcal{P}$-adic expansion

$$
x=\sum_{j=0}^{\infty} \frac{x_{j}}{m_{j+1}}, \quad 0 \leq x_{j} \leq p_{j}-1 .
$$

We denote by $Q_{\mathcal{P}}$ the set of all $\mathcal{P}$-adic rational numbers, i.e., points of the form $\frac{t}{m_{k}}, 0 \leq t \leq m_{k}$. The elements of the set $[0,1) \backslash Q_{\mathcal{P}}$ are called $\mathcal{P}$-adic irrational numbers in $[0,1]$.

We note that each $x \in Q_{\mathcal{P}}$ has two expansions, a finite one and an infinite one. We agree to consider only the finite expansion for $x \in Q_{\mathcal{P}}$. Then, to each $x \in[0,1)$ there corresponds only one sequence of integers $\left\{x_{j}\right\}_{j=0}^{\infty}, 0 \leq x_{j} \leq$ $p_{j}-1$, given by (2.2).

We also consider $\mathcal{P}$-adic expansions of integers $n \geq 0$ :

$$
n=\sum_{j=0}^{s} \alpha_{j} m_{j-1} \quad \text { with } \quad 0 \leq \alpha_{j} \leq p_{j}-1 .
$$

Now, for each $n \geq 0$ with $\mathcal{P}$-adic expansion (2.3), we define an $n$th function $\chi_{n}$ of the so-called multiplicative Vilenkin system (see [1] and [2]) by putting

$$
\chi_{n}(x):=\exp \left(2 \pi i \sum_{j=0}^{s} \frac{\alpha_{j} x_{j}}{p_{j}}\right),
$$

where numbers $x_{j}$ are given by (2.2). Note that when $p_{j}=2$ for all $j$, we obtain the Walsh system.

We consider half-open $\mathcal{P}$-adic intervals (or $\mathcal{P}$-intervals)

$$
I_{r}^{(k)}:=\left[\frac{r}{m_{k}}, \frac{r+1}{m_{k}}\right), \quad 0 \leq r \leq m_{k}-1,
$$

where the number $k=0,1,2, \ldots$ is called the rank of the interval. By $I^{(k)}$ we denote an arbitrary interval of rank $k$ and by $I_{x}^{(k)}$ the intervals of rank $k$ the closures of which $\bar{I}^{(k)}$ contain the point $x$. So, if $x \in(0,1)$, the notation $I_{x}^{(k)}$ is related to two intervals (2.5), and, for $x=0$ or 1 , to one.

We also assign the rank $k$ to a number $n$ and to a function $\chi_{n}$ if $m_{k-1} \leq$ $n<m_{k}\left(\chi_{0}\right.$ has rank 0$)$. Note that functions $\chi_{n}$ of rank $k$ are constant on the 
interval of the same rank and

$$
\int_{I^{(k-1)}} \chi_{n} \mathrm{~d} x=0
$$

We now define the double Vilenkin system on the square $K=[0,1) \times[0,1)$ by $\chi_{n, m}(x, y):=\chi_{n}(x) \times \chi_{m}(y)$. If the rank of the numbers $n$ and $m$ are equal to $k$ and $l$, respectively, we say that the pair $(n, m)$ has $\operatorname{rank}(k, l)$, and so has the function $\chi_{n, m}(x, y)$ and an interval $I^{(k, l)}:=I^{(k)} \times I^{(l)}$. The notation $I_{(x, y)}^{(k, l)}$ is referred to the interval $I_{x}^{(k)} \times I_{y}^{(l)}$ of rank $(k, l)$ the closure of which $\bar{I}^{(k, l)}$ contains the point $(x, y)$. Note that we shall use notation $I_{(x, y)}^{(k, l)}$ also for points $(x, y) \in \bar{K} \backslash K$. We denote by $\mathcal{I}$ the set of all two-dimensial $\mathcal{P}$-intervals $I^{(k, l)}$.

Functions $\chi_{n, m}(x, y)$ of rank $(k, l)$ are constant on two-dimensional $\mathcal{P}$-intervals of the same rank.

A double series in the Vilenkin system is defined by

$$
\sum_{n, m=0}^{\infty} a_{n, m} \chi_{n, m}(x, y):=\sum_{n=0}^{\infty} \sum_{m=0}^{\infty} a_{n, m} \chi_{n}(x) \cdot \chi_{m}(y),
$$

where $a_{n, m}$ are real or complex numbers. The rectangular partial sum $S_{r, s}$ of series (2.7) at a point $(x, y)$ is

$$
S_{r, s}(x, y):=\sum_{n=0}^{r-1} \sum_{m=0}^{s-1} a_{n, m} \chi_{n, m}(x, y) .
$$

Note that if $r \leq m_{k}$ and $s \leq m_{l}$, then the above partial sums are constant on each intervals of rank $(k, l)$. We say that the series (2.7) converges rectangularly to a sum $S(x, y)$ at a point $(x, y)$, and we write $\lim _{r, s \rightarrow \infty} S_{r, s}(x, y)=S(x, y)$ if

$$
S_{r, s}(x, y) \rightarrow S(x, y) \quad \text { as } \min \{r, s\} \rightarrow \infty \text {. }
$$

\section{Main result}

We give a sufficient condition for the coefficients $a_{n, m}$ of the series (2.7) to tend to zero when $n+m \rightarrow \infty$. In what follows, we suppose that the sequence $\left\{p_{i}\right\}$ is bounded by a number $P \geq 2$.

TheOREM 3.1. Suppose that a double series (2.7) with respect to Vilenkin system is rectangularly convergent everywhere on a "cross"

$$
\{a \times(0,1)\} \cup\{(0,1) \times b\},
$$

where $a$ and $b$ are $\mathcal{P}$-adic irrational points, except on a countable set $E$. Then,

$$
\lim _{n+s \rightarrow \infty} a_{n, s}=0 .
$$




\section{VALENTIN A. SKVORTSOV — FRANCESCO TULONE}

Proof. Assume that (3.1) does not hold. Then, for some $C>0$ and some sequence of indexes $\left\{n_{i}, s_{i}\right\}$, with $n_{i}+s_{i} \rightarrow \infty$ when $i \rightarrow \infty$, the inequality

$$
\left|a_{n_{i}, s_{i}}\right|>C
$$

holds for all $i$. It follows from the hypothesis of the theorem that there exist points of convergence of the series. This implies that $\lim _{n, s \rightarrow \infty} a_{n, s} \chi_{n, s}(x, y)=0$ at those points. Having in mind that $\left|\chi_{n, s}(x, y)\right|=1$, we get

$$
\lim _{n, m \rightarrow \infty} a_{n, m}=0 \text {. }
$$

From this fact it follows that in (3.2) we can consider one of the index to be bounded. Therefore, without loss of generality, we can assume that for a constant $s$ and for all $n_{i}, n_{1}<n_{2}<\cdots<n_{i}<\cdots$, the inequality

$$
\left|a_{n_{i}, s}\right|>C
$$

holds.

Let the terms of the sequence $\left\{n_{i}\right\}_{i}$ in (3.4) be of ranks $k_{i}$, respectively, and the number $s$ be of rank $l$. We can assume that $k_{1}<k_{2}<\cdots<k_{i}<\cdots$

For each $i$, we put $t_{l}^{i}(y)=\sum_{q=0}^{m_{l}-1} a_{n_{i}, q} \chi_{q}(y)$. Note that $t_{l}^{i}$ is constant on each $\mathcal{P}$-interval of rank $l$, and according to (2.6),

$$
\int_{I^{(l)}} t_{l+j}^{i}(y) \mathrm{d} y=\int_{I^{(l)}} t_{l}^{i}(y) \mathrm{d} y
$$

for any $j \geq 1$. From condition (3.4) we get

$$
\left|t_{l}^{i}(y)\right|>C
$$

on at least one interval $I^{(l)}$ of rank $l$. Indeed, if (3.6) does not hold, i.e., for all $y$ we have

$$
\left|t_{l}^{i}(y)\right|=\left|\sum_{q=0}^{m_{l}-1} a_{n_{i}, q} \chi_{q}(y)\right| \leq C,
$$

then considering $a_{n_{i}, q}$ as Fourier coefficients of $t_{l}^{i}$, we obtain in particular for $q=s$,

$$
\left|a_{n_{i}, s}\right|=\left|\int_{0}^{1} t_{l}^{i}(y) \chi_{s}(y) \mathrm{d} y\right| \leq \int_{0}^{1}\left|t_{l}^{i}(y)\right|\left|\chi_{s}(y)\right| \mathrm{d} y \leq \max _{y}\left|t_{l}^{i}(y)\right| \leq C
$$

getting a contradiction with (3.4). Taking a subsequence of $\left\{k_{i}\right\}$ if necessary, we can suppose that the interval $I^{(l)}$ is the same for each $i$ and we fix it for the further discussion. 
For a fixed $i$, consider the sum

$$
\begin{aligned}
p_{l}^{i}(x, y) & =S_{n_{i}+1, m_{l}}(x, y)-S_{n_{i}, m_{l}}(x, y) \\
& =\chi_{n_{i}}(x) \sum_{q=0}^{m_{l}-1} a_{n_{i}, q} \chi_{q}(y) \\
& =\chi_{n_{i}}(x) \times t_{l}^{i}(y) .
\end{aligned}
$$

From (3.6), it follows that there exists a double interval $I^{\left(k_{i}, l\right)}=I_{a}^{\left(k_{i}\right)} \times I^{(l)}$ of rank $\left(k_{i}, l\right)$ which intersects $a \times(0,1)$ and on which $\left|p_{l}^{i}(x, y)\right|>C$. Denote the value of $p_{l}^{i}$ on this interval by $p_{l}^{i}\left(I^{\left(k_{i}, l\right)}\right)$ and the value of $t_{l}^{i}$ on the interval $I^{(l)}$ by $t_{l}^{i}\left(I^{(l)}\right)$. Thus,

$$
\left|t_{l}^{i}\left(I^{(l)}\right)\right|=\left|p_{l}^{i}\left(I^{\left(k_{i}, l\right)}\right)\right|>C .
$$

The intervals $I^{\left(k_{i}, l\right)}$ are embedded into each other as $i$ increases and the inter$\operatorname{val} I^{(l)}$ is their common projection onto the $y$-axis.

Now, we consider the sequence $\left\{t_{l+j}^{i}\right\}_{j}$. We note that there exists a sequence of nested $\mathcal{P}$-intervals $\left\{\widehat{I}^{l+j}\right\}_{j}$ such that

$$
\left|t_{l+j+1}^{i}\left(\widehat{I}^{(l+j+1)}\right)\right| \geq\left|t_{l+j}^{i}\left(\widehat{I}^{(l+j)}\right)\right|>C, \quad j=0,1, \ldots
$$

Indeed, for $j=0$ we put $\widehat{I}^{(l)}=I^{l}$ and suppose $\widehat{I}^{(l+j)}$ has been chosen. We have

$$
t_{l+j+1}^{i}(y)=t_{l+j}^{i}(y)+\sum_{q=m_{l+j}}^{m_{l+j+1}-1} a_{n_{i}, q} \chi_{q}(y) .
$$

From (2.6), we get

$$
\int_{\widehat{I}^{(l+j)}} \sum_{q=m_{l+j}}^{m_{l+j+1}-1} a_{n_{i}, q} \chi_{q}(y) \mathrm{d} y=0 .
$$

This easily implies that at least for one of the intervals $I^{(l+j+1)} \subset \widehat{I}^{(l+j)}$ which we denote by $\widehat{I}^{(l+j+1)}$, the inequality (3.8) holds.

We formulate the next step of the proof as a separate lemma.

LEMMA 3.1. Under the assumption of Theorem 3.1 and under the above notation, suppose that for the interval $I^{(l)}$, for the sequence $\left\{n_{i}\right\}_{i}$, and for the corresponding sequence of ranks $\left\{k_{i}\right\}_{i}$, which are used in the definition of the sequence $\left\{t_{l}^{i}\right\}_{i}$, the inequality $\left|t_{l}^{i}\left(I^{(l)}\right)\right|>C>0$ holds for all $i=1,2, \ldots$ Then, for any $B, 0<B<C$ there exist two nonintersecting $\mathcal{P}$-adic intervals $\widetilde{I}^{\left(l_{1}\right)}$ and $\widehat{I}^{\left(l_{1}\right)}$, which are subintervals of $I^{(l)}$, with rank $l_{1}>l$, and a subsequence $\left\{n_{i_{m}}\right\}_{m}$ of $\left\{n_{i}\right\}_{i}$ such that

$$
\left|t_{l_{1}}^{i_{m}}\left(\widetilde{I}^{\left(l_{1}\right)}\right)\right|>B \quad \text { and } \quad\left|t_{l_{1}}^{i_{m}}\left(\hat{I}^{\left(l_{1}\right)}\right)\right|>B \quad \text { for all } i_{m} .
$$




\section{VALENTIN A. SKVORTSOV — FRANCESCO TULONE}

Proof. Take $\varepsilon=\frac{C-B}{P m_{l}}$ and according to (3.3), choose $j_{0} \geq 1$ and $i_{0}$ such that $\left|a_{n_{i}, q}\right|<\varepsilon$ for all $i>i_{0}$ and $q \geq m_{l+j_{0}}$. Then, for any such $i$ and for any $y$ we get

$$
\begin{aligned}
\left|t_{l+j_{0}+1}^{i}(y)-t_{l+j_{0}}^{i}(y)\right| & =\left|\sum_{q=m_{l+j_{0}}}^{m_{l+j_{0}+1}-1} a_{n_{i}, q} \chi_{q}(y)\right| \\
& <\varepsilon\left(m_{l+j_{0}+1}-m_{l+j_{0}}\right) \\
& =\varepsilon\left(m_{l+j_{0}} \cdot p_{l+j_{0}}-m_{l+j_{0}}\right) \\
& \leq \varepsilon P m_{l+j_{0}} \\
& =\frac{(C-B) m_{l+j_{0}}}{m_{l}} .
\end{aligned}
$$

Now, suppose that for each $j, 1 \leq j \leq j_{0}+1$, and for all intervals $I^{(l+j)} \subset I^{(l)}$ except one $\widehat{I}^{(l+j)}$, chosen as in (3.8), we have $\left|t_{l+j}^{i}\left(I^{(l+j)}\right)\right| \leq B$. Applying the last inequality with $j=j_{0}$ and having (3.5) in mind, we obtain the following estimation

$$
\begin{aligned}
\left|t_{l+j_{0}}^{i}\left(\widehat{I}^{\left(l+j_{0}\right)}\right)\right| \frac{1}{m_{l+j_{0}}} & =\left|\int t_{\left.\widehat{I}^{\left(l+j_{0}\right.}\right)} t_{l+j_{0}}^{i}(y) \mathrm{d} y\right| \\
& \geq\left|\int_{I^{(l)}} t_{l+j_{0}}^{i}(y) \mathrm{d} y\right|-\int_{I^{(l)} \backslash \widehat{I}^{\left(l+j_{0}\right)}}\left|t_{l+j_{0}}^{i}(y)\right| \mathrm{d} y \\
& -\left|\int_{I^{(l)}} t_{l}^{i}(y) \mathrm{d} y\right|-\iint_{I^{(l)} \backslash \widehat{I}^{(l)}}\left|t_{j_{0}}^{i}(y)\right| \mathrm{d} y \\
& >\frac{C}{m_{l}}-B\left(\frac{1}{m_{l}}-\frac{1}{m_{l+j_{0}}}\right) .
\end{aligned}
$$

So, $\left|t_{l+j_{0}}^{i}\left(\widehat{I}^{\left(l+j_{0}\right)}\right)\right|>\frac{m_{l+j_{0}}(C-B)}{m_{l}}+B$. Since by assumption we have $\left|t_{l+j_{0}+1}^{i}(y)\right| \leq B$ for all $y \in \widehat{I}^{\left(l+j_{0}\right)} \backslash \widehat{I}^{\left(l+j_{0}+1\right)}$, for the same $y$ we get

$$
\begin{aligned}
\left|t_{l+j_{0}+1}^{i}(y)-t_{l+j_{0}}^{i}(y)\right| & \geq\left|t_{l+j_{0}}^{i}(y)\right|-\left|t_{l+j_{0}+1}^{i}(y)\right| \\
& >\frac{m_{l+j_{0}}(C-B)}{m_{l}}+B-B \\
& =\frac{m_{l+j_{0}}(C-B)}{m_{l}} .
\end{aligned}
$$


This contradicts (3.9). So, there exists an interval $I^{(l+j)} \subset I^{(l)}, I^{(l+j)} \neq \widehat{I}^{(l+j)}$ with $j=j_{0}$ or $j=j_{0}+1$ for which $\left|t_{l+j}^{i}\left(I^{(l+j)}\right)\right|>B$. This interval together with the interval $\widehat{I}^{(l+j)}$ gives a pair $\widetilde{I}^{\left(l_{1}\right)}$ and $\widehat{I}^{\left(l_{1}\right)}$ we are looking for, with $l_{1}=l+j$.

As the rank of chosen intervals is bounded by $l+j_{0}+1$, we can take a subsequence $\left\{n_{i_{m}}\right\}$ of $\left\{n_{i}\right\}$ such that the pair $\widetilde{I}^{\left(l_{1}\right)}$ and $\widehat{I}^{\left(l_{1}\right)}$ is the same for all $i_{m}>i_{0}$. This proves the lemma.

Proceeding with the proof of the theorem, we take a sequence $C=C_{0}>$ $C_{1}>C_{2}>\cdots>\frac{C}{2}$ and applying Lemma 3.1 successively first for $C=C_{0}$ and $B=C_{1}$, then for $C=C_{1}$ and $B=C_{2}$ and so on, we double in each step the intervals obtained in the previous step. In the $q$ th inductive step we get $2^{q}$ disjoint intervals $I^{\left(l_{q}\right)}$ of the rank $l_{q}>l_{q-1}>\cdots>l_{1}>l$ and some sequence $\left\{i_{q}\right\}$ so that $n_{i_{q}}$ is a subsequence of $\left\{n_{i}\right\}$ given by (3.4) for which $\left|t_{l_{q}}^{i_{q}}\left(I^{\left(l_{q}\right)}\right)\right|>C_{q}$.

Now, we take an interval $I^{\left(k_{i_{q}}\right)}$ for which $a \in I^{\left(k_{i_{q}}\right)}$, where $k_{i_{q}}$ is the rank of $n_{i_{q}}$. Then, for any $q$ we have

$$
\left|p_{l_{q}}^{i_{q}}\left(I^{\left(k_{i_{q}}, l_{q}\right)}\right)\right|>C_{q}>\frac{C}{2} .
$$

So, we get continuum of sequences of nested intervals $\left\{I^{\left(k_{i_{q}}, l_{q}\right)}\right\}$. Hence, at least one of these sequences has a common point $\left(a, y_{0}\right) \notin E$ with $y_{0} \notin Q_{\mathcal{P}}$ and at this point, by assumption of the theorem, the series is convergent and so, for the sequence $p_{j}^{i}$ defined by (3.7) we should have $\lim _{q \rightarrow \infty} p_{l_{q}}^{i_{q}}\left(a, y_{0}\right) \rightarrow 0$. This is in contradiction to (3.10). Therefore, the assumption (3.2) is false and the theorem is proved.

\section{Application to the problem of recovering the coefficients}

Theorem 3.1 is essential for obtaining results on recovering the coefficients of convergent double Vilenkin series by generalized Fourier formulas.

A standard method for solution to the problem of recovering the coefficients in the case of many classical orthogonal series is based on reducing this problem to the one of recovering a primitive from its derivative where the derivative is defined with respect to a properly chosen derivation basis (see [6]). In the case of Vilenkin series, a suitable basis is the basis formed by $\mathcal{P}$-intervals.

Given a set function $F: \mathcal{I} \rightarrow \mathbb{R}$, we define the upper and the lower $\mathcal{P}$-derivative at a point $(x, y)$ as

$$
\bar{D}_{\mathcal{P}} F(x, y):=\limsup _{\min (k, l) \rightarrow \infty} \frac{F\left(I_{x, y}^{(k, l)}\right)}{\left|I_{x, y}^{(k, l)}\right|} \text { and } \underline{D}_{\mathcal{P}} F(x, y):=\liminf _{\min (k, l) \rightarrow \infty} \frac{F\left(I_{x, y}^{(k, l)}\right)}{\left|I_{x, y}^{(k, l)}\right|},
$$

respectively. 


\section{VALENTIN A. SKVORTSOV — FRANCESCO TULONE}

It is clear that $\bar{D}_{\mathcal{P}} F(x, y) \geq \underline{D}_{\mathcal{P}} F(x, y)$. If $\bar{D}_{\mathcal{P}} F(x, y)=\underline{D}_{\mathcal{P}} F(x, y)$, this common value is called $\mathcal{P}$-derivative $D_{\mathcal{P}} F(x, y)$. For a complex-valued set function $F=\operatorname{Re} F+i \operatorname{Im} F$, we define $\mathcal{P}$-derivative at a point $(x, y)$ as

$$
D_{\mathcal{P}} F(x, y):=D_{\mathcal{P}} \operatorname{Re} F(x, y)+D_{\mathcal{P}} \operatorname{Im} F(x, y) .
$$

The difficulties which should be overcome in solving the problem of recovering a primitive from its derivative in the multidimensional case are related to the fact that the primitive, we want to recover, is differentiable not everywhere but outside some exceptional set which is not countable in a dimension greater than one. We have to impose some continuity assumptions on the primitive at points of exceptional sets to guarantee its uniqueness. It turns out that the usual continuity with respect to the basis is not enough for this purpose and we introduce a stronger notion of continuity, which we call local Saks continuity with respect to the basis.

Definition 4.1. We say that a $\mathcal{P}$-interval function $F$ is locally $\mathcal{P}$-continuous in the sense of Saks, or briefly $\mathcal{P} S$-continuous, at a point $(x, y) \in \bar{K}$ if

$$
\lim _{k+l \rightarrow \infty} F\left(I_{x, y}^{(k, l)}\right)=0
$$

In our case, the role of the exceptional set will be played by the set $Z$ of points of the unit interval $K$ having at least one $\mathcal{P}$-rational coordinate, i.e.,

$$
Z:=\left([0,1) \times Q_{\mathcal{P}}\right) \bigcup\left(Q_{\mathcal{P}} \times[0,1)\right) .
$$

Now, we introduce a Perron type integral which solves the problem of recovering a primitive from its $\mathcal{P}$-derivative which is defined only outside the set $\mathrm{Z}$. It is a generalization of the dyadic Perron type integral $\left(P_{\mathcal{B}} S\right.$-integral $)$ introduced in [9]. Properties of this integral in more details will be studied in [10].

Definition 4.2. Let $f$ be a real point function defined at least on $K \backslash Z$. An additive $\mathcal{P} S$-continuous on $\bar{K} \mathcal{P}$-interval function $M: \mathcal{I} \rightarrow \mathbb{R}$ (resp., $m$ ) is called a $\mathcal{P} S$-major (resp., $\mathcal{P} S$-minor) function of $f$ if the lower (resp., the upper) $\mathcal{P}$-derivative satisfies the inequality

$$
\underline{D}_{\mathcal{P}} M(x, y) \geq f(x, y)\left(\operatorname{resp} . \bar{D}_{\mathcal{P}} m(x, y) \leq f(x, y)\right)
$$

for all $(x, y) \in K \backslash Z$.

Proof of the following lemma is the same as the proof of the corresponding lemma in 9] for the dyadic case.

LEMMA 4.1. Let $M$ and $m$ be a $\mathcal{P} S$-major and a $\mathcal{P} S$-minor function for a point-function $f$ on $K$. Then, for each $\mathcal{P}$-interval $I$ we have $M(I) \geq m(I)$. 
The last lemma implies that for any function $f$ we have

$$
\inf _{M}\{M(K)\} \geq \sup _{m}\{m(K)\}
$$

where "inf" and "sup" are taken over all $\mathcal{P} S$-major and $\mathcal{P} S$-minor function of $f$, respectively. This justifies the following definition.

Definition 4.3. A point-function $f: K \rightarrow \mathbb{R}$, defined at least on $K \backslash Z$, is said to be $\mathcal{P} S$-integrable on $K$ if there exists at least one $\mathcal{P} S$-major function and at least one $\mathcal{P} S$-minor function of $f$ and

$$
-\infty<\inf _{M}\{M(K)\}=\sup _{m}\{m(K)\}<\infty,
$$

where "inf" and "sup" are taken as above. The common value is called $\mathcal{P} S$-integral of $f$ on $K$ and is denoted by $(\mathcal{P} S) \int_{K} f$.

In the same way, we can define $\mathcal{P} S$-integral on any $\mathcal{P}$-interval $I$.

Directly from the definitions we get the following result which shows that the $\mathcal{P} S$-integral solves the problem of recovering the primitive from its $\mathcal{P}$-derivative in the form we need.

TheOREM 4.1. If an additive $\mathcal{P} S$-continuous $\mathcal{P}$-interval function $F$ is $\mathcal{P}$-differentiable with $D_{\mathcal{P}} F(x, y)=f(x, y)$ everywhere on $K \backslash Z$, then the function $f$ is $\mathcal{P} S$-integrable on $K$ and $F$ is its indefinite $\mathcal{P} S$-integral.

In a natural way, the definition of the $\mathcal{P} S$-integral is extended to the case of complex-valued functions.

Definition 4.4. A point-function $f: K \rightarrow \mathbb{C}$, defined at least on $K \backslash Z$, is said to be $\mathcal{P} S$-integrable on $K$ if functions $\operatorname{Re} f$ and $\operatorname{Im} f$ are integrable. In this case, the value of $\mathcal{P} S$-integral is defined as $(\mathcal{P} S) \int_{K} f:=(\mathcal{P} S) \int_{K} \operatorname{Re} f+i(\mathcal{P} S) \int_{K} \operatorname{Im} f$.

To connect the problem of recovering a primitive with the problem of recovering coefficients of the series (2.7), we introduce the following $\mathcal{P}$-interval function. For each $\mathcal{P}$-interval of rank $(k, l)$ we define

$$
\psi\left(I^{(k, l)}\right):=\int_{I^{(k, l)}} S_{m_{k}, m_{l}}(x, y) \mathrm{d} x \mathrm{~d} y .
$$

It is easy to check that $\psi$ is an additive function on algebra generated by $\mathcal{I}$. In $\mathcal{P}$-adic analysis, the function $\psi$ is referred to as the quasi-measure generated by the series (see [4]). Since the sum $S_{m_{k}, m_{l}}$ is constant on each $I^{(k, l)}$, we get

$$
S_{m_{k}, m_{l}}(x, y)=\frac{1}{\left|I^{(k, l)}\right|} \int_{I^{(k, l)}} S_{m_{k}, m_{l}}(x, y) \mathrm{d} x \mathrm{~d} y=\frac{\psi\left(I^{(k, l)}\right)}{\left|I^{(k, l)}\right|}
$$

for any point $(x, y) \in \operatorname{int} I^{(k, l)}$. 


\section{VALENTIN A. SKVORTSOV — FRANCESCO TULONE}

In fact, any additive $\mathcal{P}$-interval function $\psi$ defines a Vilenkin series for which it is the quasi-measure generated by it and (4.5) holds. So, we have one-to-one correspondence between the family of additive $\mathcal{P}$-interval functions and the family of Vilenkin series.

The equality (4.5) obviously gives a relation between $\mathcal{P}$-differentiability of $\psi$ at $(x, y)$ and convergence of the series. In particular, at least at the points $(x, y) \in$ $K \backslash Z$, we get

$$
\lim _{k, l \rightarrow \infty} S_{m_{k}, m_{l}}(x, y)=D_{\mathcal{P}} \psi(x, y),
$$

and therefore, the convergence of the series (2.7) at such points to a sum $f(x, y)$ implies $\mathcal{P}$-differentiability of the function $\psi$ at $(x, y)$ with $f(x, y)$ being the value of $\mathcal{P}$-derivative.

The next lemma demonstrates the importance of Theorem 3.1 for an application of the $\mathcal{P} S$-integral to the problem of recovering the coefficients as it gives the required continuity properties of the quasi-measure.

LEMma 4.2. If the coefficients of series (2.7) satisfy condition (3.1), then at each point $(x, y) \in K$ the quasi-measure $\psi$ is $\mathcal{P} S$-continuous, i.e., (4.2) holds everywhere on $K$.

Proof. Having in mind that for all $i<m_{k}$ and $j<m_{l}$ functions $\chi_{i, j}$ are constant on each interval $I_{(x, y)}^{(k, l)}$ of rank $(k, l)$, we have for a fixed $(x, y)$ (recall that $(x, y)$ can belong to closure of $\left.I_{(x, y)}^{(k, l)}\right)$

$$
\begin{aligned}
\left|\psi\left(I_{(x, y)}^{(k, l)}\right)\right| & \leq \int_{I_{(x, y)}^{(k, l)}}\left|S_{m_{k}, m_{l}}(t, s)\right| \mathrm{d} t \mathrm{~d} s \\
& \leq \sum_{i, j=0}^{m_{k}-1, m_{l}-1} \int_{I_{(x, y)}^{(k, l)}}\left|a_{i, j} \chi_{i, j}(t, s)\right| \mathrm{d} t \mathrm{~d} s \\
& \leq \frac{\sum_{i, j=0}^{m_{k}-1, m_{l}-1}\left|a_{i, j}\right|}{m_{k} m_{l}} .
\end{aligned}
$$

But the right-hand side expression is the arithmetic mean of the modulus of the coefficients $a_{i, j}$ and it tends to zero together with coefficients when $i+j \rightarrow \infty$.

Note that, in fact, we have proved uniform $\mathcal{P} S$-continuity of $\psi$. We also note that the above statement is not true even under assumption of convergence everywhere on $K$ of Vilenkin series if the convergence is considered with respect to regular rectangles, for example, with respect to cubes (see [3]). 
The following statement is essential for establishing that a given Vilenkin series is the Fourier series in the sense of some general integral (see, for example, [6]; a proof in the one-dimensional version can be found in [2, Th. 3.1.8]).

Proposition 4.1. Let some integration process $\mathcal{A}$ be given which produces an integral additive on $\mathcal{I}$. Assume a series of the form (2.7) is given. Let a $\mathcal{P}$-interval function $\psi$ be the quasi-measure generated by this series and (4.5) holds. Then this series is the Fourier series of an $\mathcal{A}$-integrable function $f$ if and only if $\psi(I)=(\mathcal{A}) \int_{I} f$ for any $\mathcal{P}$-interval $I$.

In view of (4.6) and the above proposition, in order to solve the coefficient problem it suffices to show that the quasi-measure $\psi$ generated by the Vilenkin series is the indefinite $\mathcal{P} S$-integral of its $\mathcal{P}$-derivative which exists at least on $K \backslash Z$.

Finally, using all the above results, we get

Theorem 4.2. If a two-dimensional series (2.7) is rectangular convergent to a sum $f$ everywhere in $K \backslash Z$, then $f$ is $\mathcal{P} S$-integrable on $K$ and the coefficients of the series are $\mathcal{P} S$-Fourier-Vilenkin coefficients of $f$.

Proof. Take any $(a, b) \in K \backslash Z$. Then intersection of the cross $\{a \times[0,1]\} \cap$ $\{[0,1] \times b\}$ with $Z$ is countable, and by Theorem 3.1, condition (3.1) holds. Then, by Lemma 4.2, the quasi-measure $\psi$ generated by the series (2.7) is $\mathcal{P} S$-continuous everywhere on $K$. Moreover, the equality (4.6) implies

$$
\lim _{k, l \rightarrow \infty} S_{m_{k}, m_{l}}(\mathbf{x})=D_{\mathcal{P}} \psi(x, y)=f(x, y)
$$

everywhere on $K \backslash Z$. Then, application of Proposition 4.1 and Theorem 4.1 to the real and imaginary parts of functions $\psi$ and $f$ completes the proof.

\section{REFERENCES}

[1] AGAEV, G. N.-VILENKIN, N. YA.-DZHAFARLI, G. M.-RUBINSTEIN, A. I.: Multiplicative System of Functions and Harmonic Analysis on Zero-Dimensional Groups. Èlm, Baku, 1981. (In Russian)

[2] GOLUBOV, B.-EFIMOV, A.-SKVORTSOV, V.: Walsh Series and Transforms: Theory and Applications. Nauka, Moscow 1987; Translated: Mathematics and its Applications (Soviet Series), Vol. 64, Kluwer Academic Publishers Group, Dordrecht, 1991.

[3] PLOTNiKOV, M. G.: Recovery of the coefficients of multiple Haar and Walsh series, Real Anal. Exchange 33 (2007), 291-308.

[4] SCHIPP, F.-WADE, W. R.-SIMON, P.-PAL, J.: Walsh Series: An Introduction to Dyadic Harmonic Analysis. Adam Hilger Publ., Ltd., Bristol, 1990.

[5] SKVORTSOV, V. A.: On coefficients of convergent multiple Haar and Walsh series, Vestnik Moskov. Univ., Ser. I Mat. Mekh. 6 (1973), 77-79. (In Russian); English transl.: Moscow Univ. Math. Bull. 28 (1974), 119-121. 


\section{VALENTIN A. SKVORTSOV — FRANCESCO TULONE}

[6] Henstock-Kurzweil type integrals in $\mathcal{P}$-adic harmonic analysis, Acta Math. Acad. Paedagog. Nyházi. (N.S.) 20 (2004), 207-224.

[7] SKVORTSOV, V. A.-TULONE, F.: Perron type integral on compact zero-dimensional Abelian groups, Vestnik Moskov. Univ., Ser. I Mat. Mekh. 63 (2008), 37-42. (In Russian); English transl.: Moscow Univ. Math. Bull. 63 (2008), 119-124.

[8] Kurzweil-Henstock type integral on zero-dimensional group and some of its applications, Czechoslovak Math. J. 58 (2008), 1167-1183.

[9] Multidimensional dyadic Kurzweil-Henstock- and Perrop-type integral in the theory of Haar and Walsh series, J. Math. Anal. Appl. 421 (2015), 1502-1518.

[10] TUlONE, F.-SKVORTSOV, V. A.: Multidimensional P-adic integral in some problems of harmonic analysis, Minimax Theory Appl. 2 (2017), 153-174.

Received November 11, 2016

Valentin A. Skvortsov

Department of Mathematics

Moscow State University

Moscow 119991

RUSSIA

E-mail: vaskvor2000@yahoo.com

Francesco Tulone

Department of Mathematics and

Computer Sciences

Palermo University

90123 Palermo

ITALY

E-mail: francesco.tulone@math.unipa.it 